\title{
A NOTE ON MAXIMAL LOCALLY COMPACT SEMIGROUPS
}

JAMES W. STEPP

1. Introduction. Let $S$ be the class of semigroups, and let $\&$ be those members of $S$ that are locally compact. We use the notation $S<T$ $(S, T \in S)$ to mean $S$ is a subsemigroup of $T$. By $S \triangleleft T$ we mean both $S<T$ and $S$ is dense in $T$. Finally, we introduce the notion of a maximal semigroup; an element $S$ in $\mathscr{L}$ is said to be maximal in $\mathfrak{L}(\mathcal{S})$ if $S$ is closed in all $T \in \mathscr{L}(\mathcal{S})$ with $S<T$.

We first show that for each $S$ in $\mathscr{L}$ there exists a $T$ in $\mathscr{L}$ such that $T$ is maximal in $\mathscr{L}$ and $S \triangleleft T$. Also, we show that there exists a noncompact $S \in \mathscr{L}$ that is maximal in $\mathcal{S}$. In fact, it will be shown that the semigroups described by Hoffman in [1] and those of the Mostert Type [2] are maximal in $\delta$.

II. Existence theorem. In this section we prove the existence theorem and give most of the definitions and notation that will be used throughout this note.

If $S \triangleleft T$, then $T$ will be called an $\mathrm{M}(S)$ semigroup if $T$ is maximal in $\mathcal{S}$, and $T$ will be called an $\operatorname{LCM}(S)$ semigroup if $T$ is in $\mathscr{L}$ and $T$ is maximal in $\mathcal{L}$.

For the existence theorem we will denote a semigroup by a triple $(S, F, m)$, where $S$ is the set, $F$ is the topological structure and $m$ is the multiplication function.

Existence Theorem. If $(S, F, m) \in \mathfrak{L}$, then there exists an $\operatorname{LCM}(S)$ semigroup.

Proof. Let $d=\inf \{|N| \mid N \subseteq S, \bar{N}=S\}$, where $|N|$ denotes the cardinal number of $N$. We make use of the facts [3, pp. 50-52] that $|S| \leqq 2^{2^{d}}$ and if $S \triangleleft T$, then $|T| \leqq 2^{2^{d}}$.

Let $Y$ be a set with $2^{2^{d}}<|Y|$ and $S \subseteq Y$, let $X_{1}$ be the set of subsets of $Y$, let $X_{2}$ be the set of subsets of $X_{1}$, let $X_{3}$ be the set of functions from $Y \times Y$ into $Y$, and finally, for $f$ in $X_{1} \times X_{2} \times X_{3}$ let $\hat{f}(3)$ be $f(3)$ restricted to $f(1) \times f(1)$, where $f=(f(1), f(2), f(3))$.

Let $C$ be the collection of all $f$ in $X_{1} \times X_{2} \times X_{3}$ so that $f(3)(f(1)$ $\times f(1)) \subseteq f(1),(f(1), f(2), \hat{f}(3)) \in \mathscr{L}$ and $(S, F, m) \triangleleft(f(1), f(2), \hat{f}(3))$. Since there exists a $g$ in $X_{1} \times X_{2} \times X_{3}$ with $g(1)=S, g(2)=F$ and 1967.

Presented to the Society, November 18, 1967; received by the editors October 18, 
$\hat{g}(3)=m, C \neq \varnothing$. Partially order $C$ by: if $f, l$ are in $C$, then $f \leqq l$ if and only if $(f(1), f(2), \hat{f}(3))<(l(1), l(2), \ell(3))$.

Pick a maximal chain $D$ from $C$, and let $R=\bigcup\{f(1) \mid f \in D\}$. Let $a=\{U \subseteq R \mid U \cap f(1) \in f(2)$ for all $f$ in $D\}$. Define $n: R \times R \rightarrow R$ by $n(x, y)=f(3)(x, y)$ where $f \in D$ and $f$ is such that $(x, y) \in f(1) \times f(1)$. By $[4$, p. 131], $(f(1), f(2))$ is an open subspace of $(R, a)$ for each $f$ in $D$. Since $D$ is a chain and $(f(1), f(2))$ is open in $(l(1), l(2))$ whenever $f \leqq l,(R, a)$ is locally compact and Hausdorff. Since $(R, a)$ is a locally compact Hausdorff space, $\{U \subseteq R \times R \mid U \cap(f(1) \times f(1))$ is open in $f(1) \times f(1)$ for all $f$ in $D\}$ is the product topology on $R \times R$. By $[4$, p. 132], $n$ is continuous. Therefore, $(R, Q, n) \in \mathscr{L}$ and $(S, F, m)$ $\triangleleft(R, Q, n)$.

Let $\left(T, F_{2}, p\right)$ be an element of $\mathscr{L}$ with $(R, Q, n) \triangleleft\left(T, F_{2}, p\right)$. Then, since $|T| \leqq 2^{2^{d}}<|Y|$ and $R \subset Y$, there exists a one-to-one function $\&$ from $T$ into $Y$ so that $h \mid R$ is the identity map on $R$. Clearly, there exists a topological structure $F_{3}$ and a $p_{1} \in X_{3}$ so that $(R, a, n)$ $<\left(h(T), F_{3}, \hat{p}_{1}\right)$ and $\left(h(T), F_{3}, p_{1}\right) \in \mathcal{L}$. Let $k$ be an element of $C$ with $k(1)=h(T), k(2)=F_{3}$ and $\hat{k}(3)=\hat{p}_{1}$. Since $D$ is a maximal chain and $f \leqq k$ for all $f \in D, k \in D$. Thus $(k(1), k(2), \hat{k}(3))<(R, a, n)$ and $k(1) \subseteq R \subseteq k(1)=h(T)$. This completes the proof.

The following examples show that an LCM(S) semigroup need not be unique. (1) Let $T_{1}$ be the nonnegative real numbers with the usual topology and multiplication. In $\S 3$, we show $T_{1}$ is an $\operatorname{LCM}(S)$ semigroup for any $S \triangleleft T_{1}$. (2) In [5], Horne describes a locally compact semigroup with an open dense group $P$, where $P$ is iseomorphic with the positive real numbers with the usual topology and ordinary multiplication and $S-P$ is not a compact group. By the existence theorem, there exists an $\operatorname{LCM}(S)$ semigroup $T_{2}$ and clearly, $T_{2}$ is also a $\operatorname{LCM}(P)$ semigroup.

III. $\mathrm{M}(S)$ semigroups. In this section we describe a collection of $\mathrm{M}(S)$ semigroups. In particular, we show why those semigroups in [1] and the ones of the Mostert Type [2] are $\mathrm{M}(S)$ semigroups.

THEOREM. Let $S$ be an element of $S$, and suppose there exists a $t$ in $S$ that has a compact neighborhood. Let $S$ be such that for each $T$ in $S$ with $S \triangleleft T$ there exists a group of homeomorphisms $G$ acting on $T$ with the following properties: (1) $g(S)=S$ for all $g \in G$, and (2) if $U$ is an open set in $T$ with $t \in U$ and $s \in T$, then there exists a $g \in G$ with $g(s) \in U$. Then $S \in \mathcal{L}$ and $S$ is a $\mathrm{M}(S)$ semigroup for any $S_{1} \in \mathcal{S}$ with $S_{1} \triangleleft S$.

Proof. Let $T$ be an element of $\delta$ with $S \triangleleft T$. Then, since $S$ has a compact neighborhood at $t$, there exists a set $U$ (open in $T$ ) so that $t \in U \subseteq S$. 
Let $x$ be an element of $T$. Then by (2) there exists an $h \in G$ so that $h(x) \in U \subseteq S$. By $(1), h^{-1}(h(x))=x \in h^{-1}(S)=S$.

CoROllary. Let $S$ be an element of $\mathcal{S}$ which contains an dense group $G$ and a zero. If $S$ has a compact neighborhood of zero, then $S \in \mathcal{L}$ and $S$ is a $\mathrm{M}\left(S_{1}\right)$ semigroup for any $S_{1}$ in $S$ with $S_{1} \triangleleft S$.

Using the above corollary and the fact [1] that a compact ideal can be regarded as a zero, we have the following corollary.

Corollary. Let $S_{1}, \cdots, S_{n}$ be elements of $\mathfrak{L}$ which contain dense groups $G_{1}, \cdots, G_{n}$ respectively and compact ideals $I_{1}, \cdots, I_{n}$ respectively. Then $\chi_{i=1}^{n} S_{i}$ is a $\mathrm{M}(S)$ semigroup for any $S$ in $\delta$ with $S \triangleleft X_{i=1}^{n} S_{i}$.

TheOREM. Let $S$ be a $\mathrm{M}(S)$ semigroup with a 1 , and let $C$ be a compact group. Then $S \times C$ is a $\mathrm{M}\left(S_{1}\right)$ semigroup for any $S_{1} \in S$ with $S_{1} \triangleleft S \times C$.

Proof. Let $T$ be in $S$ with $S \times C \triangleleft T$, and let $x \in T$. Then there exists a net $\{(g(\alpha), h(\alpha)) \mid \alpha \in A\}$ that is contained in $S \times C$ and converges to $x$. Since $C$ is compact, there exists a $g$ in $C$, a directed set $B$ and a function $\theta$ from $B$ into $A$ so that $\{h(\theta(\beta)) \mid \beta \in B\}$ is a subnet of $\{h(\alpha) \mid \alpha \in A\}$ that converges to $g$. Then $\{(g(\theta(\beta)), h(\theta(\beta))) \mid \beta \in B\}$ converges to $x$ and $\{(1, h(\theta(\beta))) \mid \beta \in B\}$ converges to $(1, g)$. Since $C$ is a compact group, $\left\{\left(1, h(\theta(\beta))^{-1}\right) \mid \beta \in B\right\}$ converges to $\left(1, g^{-1}\right)$. Therefore $\left\{(g(\theta(\beta)), \quad h(\theta(\beta)))\left(1, h(\theta(\beta))^{-1}\right) \mid \beta \in B\right\}$ converges to $x\left(1, g^{-1}\right)$. This implies $x\left(1, g^{-1}\right) \in \mathrm{Cl}(S \times\{1\})=S \times\{1\}$. Therefore, $x \in S \times\{g\} \subseteq S \times C$. This completes the proof.

\section{REFERENCES}

1. K. H. Hofmann, Locally compact semigroups in which a subgroup with compact complement is dense, Trans. Amer. Math. Soc. 106 (1965), 19-51.

2. T. S. Wu, Locally compact semigroups with dense maximal subgroups, Trans. Amer. Math. Soc. 113 (1964), 151-168.

3. M. A. Maurice, Compact ordered spaces, Math. Centre Tracts, Mathematish Centrum, Amsterdam, 1964.

4. J. Dugundji, Topology, Allyn and Bacon, Boston, Mass., 1965.

5. J. G. Horne, Jr., The boundary of a one-parameter group in a semi-group, Duke Math. J. 31 (1964), 109-117.

6. J. L. Kelley, General topology, Van Nostrand, Princeton, N. J., 1955.

UNIVERSITY OF KENTUCKY 